\title{
Dynamic Coal Logistics Facility Location under Demand Uncertainty
}

\author{
Jiang Wu, Jingfeng Li \\ School of Economics and Management, Shanxi University, Taiyuan, China \\ Email: wujiang198908@126.com
}

Received April 2014

\begin{abstract}
In this paper, dynamic facility location and supply chain planning are studied through minimizing the costs of facility location, path selection and transportation of coal under demand uncertainty. The proposed model dynamically incorporates possible changes in transportation network, facility investment costs, operating cost and changes in facility location. In addition, the time variation and the demand uncertainty for coal in each period of the planning horizon is taken into account to determine the optimal facility location and the optimal production volumes. Computational process and results are presented for the model in a java language. Also, an empirical case study in Shanxi province is conducted in order to investigate the dynamic effects of traffic congestion and demand uncertainty on facility location design and total system costs.
\end{abstract}

\section{Keywords}

\section{Supply Chain Design, Facility Location, Path Planning, Networks}

\section{Introduction}

In the volatile market environment, to ensure the coal supply chain (SC) in the Chinese major energy province of Shanxi stability is an important part of ensure the stable supply of energy in china. Logistics investment and operations cost was huge to improve the coal supply capacity and efficiency. And these expenses keep growing as the transformation of economic development in the province. However A significant portion of these logistics expenses are attributed to suboptimal logistics network planning and unreliable day-to-day operations [1].

Supply chain network design (SCND) has raised significant interests among both researchers and practitioners. Several investigations have been done about facility location models [2] [3]. Facility location models try to answer the following questions: How many facilities should be open? Where each facility should be located? What is the allocation? Which set of collection centers should be opened and operated? In these models, the main objective is to determine decision variables to minimization the total cost generally. However, strategic planning problems as the allocation of demands and the sites of facilities in distribution networks are often multi-objective in nature. On the other hand, some researchers investigated uncertainty which is the major sources of vagueness in facility location model. The demand uncertainty is defined as inexact forecasting demand or as volatility demands [4]. With the fluctuations of the global economy in recent years, the effect on the coal industry which has 
much close relationship with the economic status is profound enough. To our knowledge, most of authors have not taken into account the simple and direct solution for the model with the uncertain parameters. Thus, it is valuable to examine integrated models including multi-objective models with uncertain parameters.

In this paper, a facility location model is proposed for coal supply chain in Shanxi. The model is designed for mines, demand markets, and collection centers. The goal is to know how many and which collection centers should be open, and the coal in which quantities should be stock in them. The objective function is to minimize the total cost. In this paper, the model is developed to be a dynamic model by considering the uncertain demand in different periods. Then, the model is solved by two methods including Java language and the list method in operations research. Finally, computational results are discussed and analyzed with a case. This research is among the first attempt to solve the facility location problems by java programming.

The organization of the paper as follows: Section 2, a description of the problem and the integer programming mathematical model are provided. Mathematical model solving process in java language is in Section 3 with a case study. Section 4 concludes the paper and presents guidelines for future researches.

\section{Literature Review}

The problem of locating facilities is not new to the operations research community, the challenge of where to best site facilities has inspired a rich, colorful and ever growing body of literature. Early studies stem from facility location problems, which can be traced back to about a century ago [5]. Traditional location models, e.g., the p-median and incapacitated facility location models (UFLP), simply assume that the long-term operational costs can be captured by one-period transportation operations from service facilities to customer locations. See Daskin (2001) and Drezner (1995) for a review on these developments [6] [7]. As the global competition increased in the late 1970s, researchers tried to use location models to address strategical planning issues in logistics systems where complex transportation and multi-objective problems are involved. Farahani, SteadieSeifi and Asgari (2010) reviewed the various criteria and objectives and their solution methods used in facility location problem, which plays a critical role in the SCND problem. The distributor's task is to select suppliers that will deliver to each warehouse and to assign warehouses to customers so that the requirements of all parties are met to the greatest extent possible. Huijun et al. (2008) presented a bi-level programming model for location of logistic distribution centers by considering benefits of customers and logistics planning departments. They think divide the planners and customers into different levels can analyse the different and even conflict objectives at the same time in the decision-making process and reflect the practical problem better. For the more, it can also represent the mutual-action between the system managers and the customers. Altiparmak et al. (2006) presented a SC model with three objectives: namely minimization of the total cost, maximization of the service level and maximization of the capacity utilization balance for distribution centers. They proposed a solution procedure based on a genetic algorithm to obtain the set of Pareto optimal solution and implemented different weight approaches to enable the decision maker for evaluating a greater number of alternative solutions of their model [8].

The use of uncertainty in SCND models is a natural extension of a deterministic approach because all the model parameters, in practice, are not certain. This consideration results in the more realistic problems. So that uncertainties from both customer's demand generation and supply services reliability have been recognized and investigated [9]. However, most existing studies assume that the demand is deterministic and known. Such assumptions avoid dealing with change in demand that involve high nonlinearities and complex interdependence, and thus allow for a relatively simple formulation of the network design problem. However, these assumptions may not always reflect the reality, particularly in today's complex coal supply chain and intermodal transportation environment (Minner 2003). Factors such as demand (volume, regional distribution) may change, while decisions about facility locations are made on a long-term basis. Depots distribution centers and transshipment points once established shall be used for a couple of period, relocation and/or redimensioning of facilities can be quite costly. So without careful consideration of integrated shipment expedition and inventory management strategies, a planned logistics network is not likely to provide proper redundancy to sustain smooth operations.

However, all the aforementioned researches assumed that the operational characteristics of, and hence the design parameters for, the logistics network were deterministic. Fleischmann et al. (2000) also pointed out that uncertainty is an important characteristic of product flow so this issue seems to deserve additional research effort. More comprehensive quantitative results would be useful, concerning the impact of uncertainty on network design and the appropriateness of traditional approaches for capturing this element. In order to handle the prob- 
lem with such stochastic aspects, Liste and Dekker (2005) proposed a stochastic programming approach by which a deterministic location model for product recovery network design was extended to account for the uncertainties [10]. However, this research for network design under uncertainty can only address a modest number of scenarios for the uncertain problem parameters. Santoso et al. (2005) applied a recently proposed sampling strategy, which is based on crude Monte Carlo samples. But the major disadvantage of such a sampling approach is that some computational effort might be wasted on optimizing when the approximation is not accurate. Moreover, none of the aforementioned researches considered the dynamic aspect in an uncertain logistics network context [11].

Considering all these, the main contributions of this research are (a) dynamic planning for coal supply chain design and facility location, (b) minimization the costs considering the (c) demand uncertainty modeling.

\section{Model Development}

\subsection{Network Description}

In this paper, a dynamic facility location model is proposed to cope with these issues for the coal supply network in Shanxi. Figure 1 shows the network which includes mine, logistics centers, and demand markets. Through the transfer and distribution function of the logistics centers we can enlarge the circulation infrastructure function, and reduce logistics costs, improve the logistics efficiency. So we defined parameter $\alpha \in\{0,1\}$ as the expense relationship between the direct transportation and the transportation though the logistics centers. As the figure presentation, The realistic routes linking the all kinds of notes (mine, logistics centers, demand markets) are varied according to the different period (represent the uncertainty demand).In a dynamic version of the attempted network design, for every facility a close or open option is available at the beginning of every period $\mathrm{t}=$ $1, \ldots, \mathrm{T}$, where $\mathrm{T}$ denotes a given planning horizon. The cost associated with such relocation of facilities is taken into account. The objective is to know how many and which mine and logistics centers should be choose under the different demand.

The following assumptions are made in the network configuration:

- Transform selected coal mine does not produce cost.

- Locations of demand markets are fixed.

- Transportation cost per $\mathrm{km}$ is fixed.

- Potential locations of mine and logistics centers are known in advance.

- The capacities of logistics centers are unlimited.

- The capacities of mine are limited.

\subsection{Mathematical Programming Model}

The network can be formulated as a mixed-integer nonlinear programming model. Sets, parameters, and decision variables are defined as summarized in Table 1.

The decisions of the dynamic logistics network configuration include deciding whether to open or close the logistics centers at each potential depot and the quantities of coal that are to be shipped in the transportation links at each time period.

The decision variables of the model are as follows:

Time Period $t$

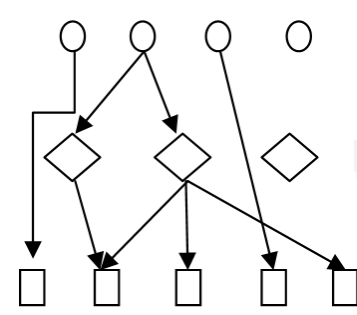

Figure 1. A depiction of the dynamic logistics network structure.
Time Period $t+2$

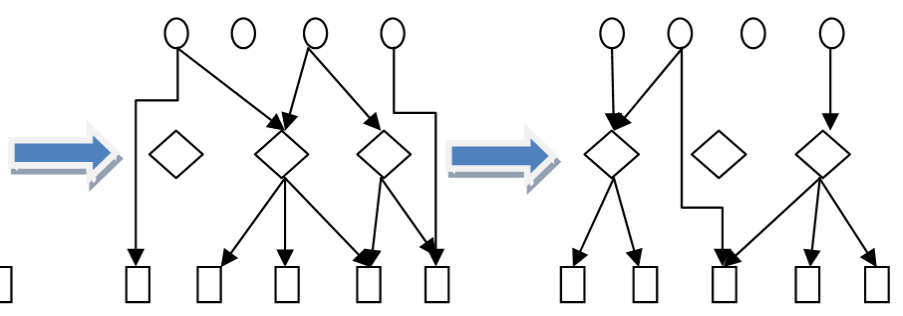


Table 1. Notation in Section 2.

\begin{tabular}{|c|c|c|}
\hline \multirow[t]{4}{*}{ Set: } & $\mathrm{I}=\{1, \ldots, \mathrm{i}\}$ & Set of potential mine \\
\hline & $J=\{1, \ldots, j\}$ & Set of potential logistics centers \\
\hline & $\mathrm{K}=\{1, \ldots, \mathrm{k}\}$ & Set of customers \\
\hline & $\mathrm{T}=\{1, \ldots, \mathrm{t}\}$ & Set of time periods in a given planning horizon \\
\hline \multirow[t]{11}{*}{ Parameters: } & $d_{k}$ & Demand of customer $k$ during time period \\
\hline & $s_{i k}$ & The distance between location $i$ and $k$ generated based on the Euclidean method \\
\hline & $q_{i k}^{t}$ & Quantity of coal from mine $i$ to demand market $k$ directly during time period $t$. \\
\hline & $S_{i j k}$ & The distance from location $i$ to $k$ via location $j$ generated based on the Euclidean method. \\
\hline & $q_{i j k}^{t}$ & Quantity of coal from mine $i$ to demand market $k$ via logistics center $j$ during time period $t$. \\
\hline & $B$ & Transportation cost per km (freight rates). \\
\hline & $\alpha$ & Expense coefficient of the transportation which via the logistic center. $A \in\{0,1\}$ \\
\hline & $f_{j}^{t}$ & Operating cost of a logistics center open at potential location $j$ during time period $t$ \\
\hline & $g f_{j}^{t}$ & Potential location $j$ at time period $\mathrm{t}$ which is closed at time period $\mathrm{t}-1$ \\
\hline & $p f_{j}^{t}$ & $\begin{array}{l}\text { Fixed cost for closing a logistics center at potential location } j \text { at time period } t \text { which is opened } \\
\qquad \text { at time period } t-1\end{array}$ \\
\hline & $f f_{j}^{t}$ & Capacity of logistics center $j$ for handling coal if forward processing facility is built at time period $t$ \\
\hline
\end{tabular}

$$
x_{j}^{t}= \begin{cases}1 & \text { if logistics centre is open at depot } j \text { at the beginning of time period } t \\ 0 & \text { otherwise }\end{cases}
$$

The objective function is formulated in the following equation:

$$
\begin{array}{lc}
\min \sum_{t \in T} \sum_{i \in I} \sum_{k \in K} B s_{i k} q_{i k}^{t}+\sum_{t \in T} \sum_{i \in I} \sum_{j \in J} \sum_{k \in K} \alpha B s_{i j k} q_{i j k}^{t}+\sum_{t \in T} \sum_{j \in J} f_{j}^{t} x_{j}^{t} \\
+\sum_{t \in T}\left[\sum_{j \in J} g f_{j}^{t}\left(1-x_{j}^{t-1}\right) x_{j}^{t}+\sum_{j \in J} p f_{j}^{t} x_{j}^{t-1}\left(1-x_{j}^{t}\right)\right] \\
\sum_{i \in I} q_{i k}^{t}+\sum_{i \in I} \sum_{j \in J} q_{i j k}^{t} \geq d_{k}^{t} \geq 0 & \forall k \in K, t \in T \\
q_{i k}^{t} \geq 0 & \forall i \in I, k \in K, t \in T \\
x_{j}^{t} \in\{0,1\} & \forall j \in J, t \in J, k \in K, t \in T \\
x_{j}^{0}=0 & \forall j \in J
\end{array}
$$

In the proposed deterministic model, the objective function (1) seeks to minimize the total investment and operational costs in the dynamic logistics network. The first and second parts show the transportation cost, respectively. The third part represents the operating cost of the logistic centers which are open in time period $t$. The forth part means the cost associated with the relocation of the logistics centers. Constraint (2) ensures that the total coal demands by customers are met. Constraint (3) and (4) enforce the non-negativity of the flow variables corresponding to the coal transportation. Constraint (5) enforce the binary restriction on the location decision 
variables. Constraint (6) means that all of the potential locations of the logistics centers are closed in the start of the whole time.

\section{Solution Method}

In this paper, a particular programming language named java will be used. Very close in the grammar of Java language and $\mathrm{C}$ language and $\mathrm{C}++$ language, so that most programmers are very easy to learn and use. On the other hand, Java discarded many useless, indigestion or confused characteristics in $\mathrm{C}++$, such as mandatory type operator overloading, multiple inheritance, automatic conversion. Moreover, The Java language does not use pointers, but references. Java is different from the general implementation of computer language and the interpretation and execution of computer language [12]. The source code is compiled into a binary bytecode, and then relies on the virtual machine on different platforms to interpret and execute the bytecode. In order to achieve "one compiled, everywhere implementation". So it can provide a ubiquitous open structure and transfer information between multi-platform in the low cost way. The excellent characteristics of Java language, which makes Java application unparalleled robustness and reliability, also reduced application maintenance costs.

Depending on this consideration, we employ java to solve this problem. Since the uncertain demands makes the problem much more difficult to be solved. We divide the solving process into three steps.

\section{The Minimum Cost Problem with Unlimited Yield in One Period}

In this step we assume coal production capacity is unlimited means that all of the demand can be satisfied by any one of the coal mine in order to prevent change the coal mine with minimum cost path due to the capacity constraints.

As mentioned before we definition parameter $\alpha \in\{0,1\}$ expense coefficient of the transportation which via the logistic center. So we will have two kinds of freight rates: $B$ and $\alpha B$.

Because of the freight is far greater than the fixed cost and operation cost in coal logistics, the minimum cost solution turned into choose the path which has the minimum product of the distance and the freight rates. As shown in Figure 2, the results can be divided into 2 groups which are Group A, Group B. For group A, all the data of the nodes (name, quantity, and species) and the path (distance, freight rates) are input to the system. In the group $B$, sentences are used to determine whether all paths must be through the distribution center, and calculate the corresponding result.

\section{Determine Whether the Minimum Cost Path through the Distribution Station}

As we known the freight rate (transportation cost per $\mathrm{km}$ ) is different between the direct transport and indirect transport. So we must choose the better solution through compare the costs of these two kinds of transportation. The main code of compare procedure is shown in Figure 3.

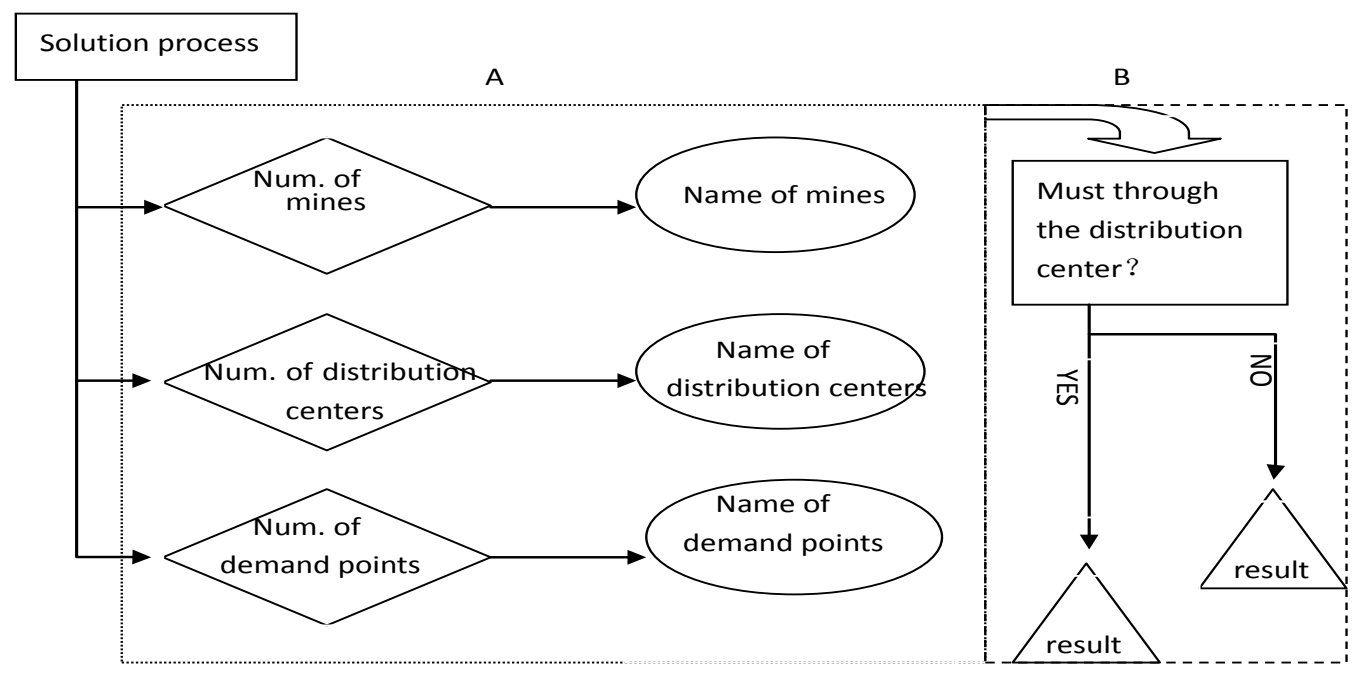

Figure 2. A schematic diagram of the best path extraction algorithm. 


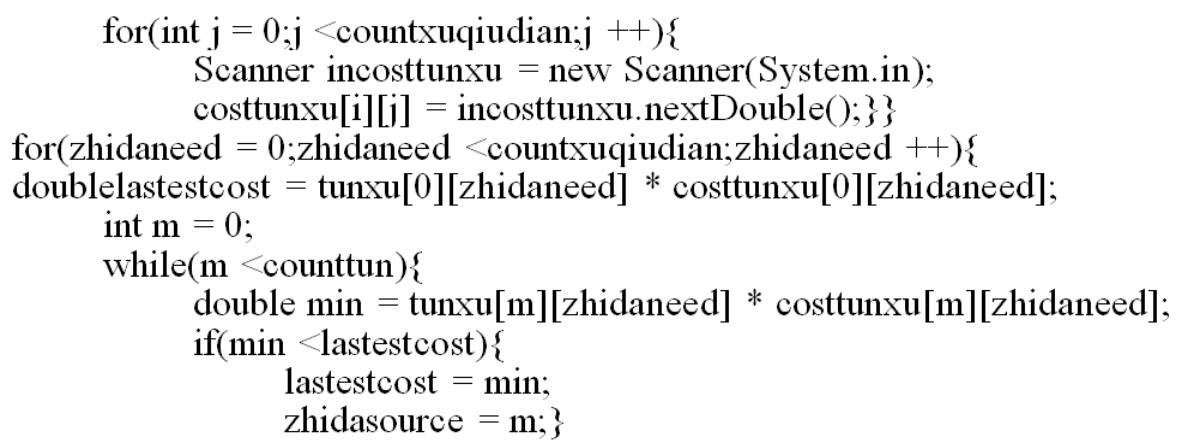

Figure 3. The compare and choose procedure.

\section{Conclusions and Future Work}

In this paper, dynamic facility location and supply chain planning were discussed, optimization of facility location and total transportation costs under demand uncertainty were investigated. Furthermore, the proposed model was coded and calculated by Java language and verified by examples. The analyses of the results from the examples showed the flexibility of the model in capturing the features of real-world problems. The model was shown to be capable of determining the location of facilities and supply coal volumes flown in the network considering the changes in parameters to satisfy the uncertain demands. This can also be verified by the results from the case study. The results from case showed that the changes in the model parameters during the periods of the planning horizon significantly affect the decisions regarding the location and supply chain planning.

Considering the uncertainty and time-variability in other parameters of the model can be a subject for future researches. In this paper, the operating cost and relocation cost are considered to be too small to effect the path selection. However, in the real world, established a logistics center often causes a great cost if the scale of center is big enough. To be more specific, we neither discussed nor developed a solution method included these factors.

\section{Acknowledgements}

This work was supported by [The Soft Science Project of Shanxi] under Grant [2013041014-02].

\section{References}

[1] Amin, S.H. and Zhang, G. (2012) A Multi-Objective Facility Location Model for Closed-Loop Supply Chain Network under Uncertain Demand and Return. Applied Mathematical Modelling, 32, 133-234.

[2] Aikens, C.H. (1985) Facility Location Models for Distribution Planning. European Journal of Operational Research, 22, 263-279. http://dx.doi.org/10.1016/0377-2217(85)90246-2

[3] Altiparmak, F., Gen, M., Lin, L. and Paksoy, T. (2006) A Genetic Algorithm Approach for Multi-Objective Optimization of Supply Chain Networks. Computers \& Industrial Engineering, 51, 196-215. http://dx.doi.org/10.1016/j.cie.2006.07.011

[4] Fahimnia, B., Farahani, R.Z., Marian, R. and Luong, L. (2012) A Review and Critique on Integrated Production-Distribution Planning Models and Techniques. Journal of Manufacturing Systems, 85, 105-119.

[5] Weber, G. (1957) Intramolecular Transfer of Electronic Energy in Dihydro Diphosphopyridine Nucleotide.

[6] Daskin, M.S. (2011) Network and Discrete Location: Models, Algorithms, and Applications. John Wiley \& Sons.

[7] Drezner, Z. (1995) Facility Location: A Survey of Applications and Methods. Springer Verlag. http://dx.doi.org/10.1007/978-1-4612-5355-6

[8] Altiparmak, F., Gen, M., Lin, L. and Paksoy, T. (2006) A Genetic Algorithm Approach for Multi-Objective Optimization of Supply Chain Networks. Computers \& Industrial Engineering, 51, 196-215. http://dx.doi.org/10.1016/j.cie.2006.07.011

[9] Snyder, L.V. (2006) Facility Location under Uncertainty: A Review. IIE Transactions, 38, 547-564. http://dx.doi.org/10.1080/07408170500216480 
[10] Listeş, O. and Dekker, R. (2005) A Stochastic Approach to a Case Study for Product Recovery Network Design. European Journal of Operational Research, 160, 268-287. http://dx.doi.org/10.1016/j.ejor.2001.12.001

[11] Santoso, T., Ahmed, S., Goetschalckx, M. and Shapiro, A. (2005) A Stochastic Programming Approach for Supply Chain Network Design under Uncertainty. European Journal of Operational Research, 167, 96-115.

http://dx.doi.org/10.1016/j.ejor.2004.01.046

[12] Lin, L., Gen, M. and Wang, X. (2009) Integrated Multistage Logistics Network Design by Using Hybrid Evolutionary Algorithm. Computers \& Industrial Engineering, 56, 854-873. http://dx.doi.org/10.1016/j.cie.2008.09.037 